This item was submitted to Loughborough's Research Repository by the author.

Items in Figshare are protected by copyright, with all rights reserved, unless otherwise indicated.

\title{
Partial cancellation of shaking force harmonics by cam modification
}

PLEASE CITE THE PUBLISHED VERSION

http://dx.doi.org/10.1243/0954406971522024

PUBLISHER

(c) IMechE / Professional Engineering Publishing

VERSION

VoR (Version of Record)

LICENCE

CC BY-NC-ND 4.0

REPOSITORY RECORD

Chiou, Shen-Tarng, and T.H. Davies. 2019. "Partial Cancellation of Shaking Force Harmonics by Cam Modification". figshare. https://hdl.handle.net/2134/4564. 
This item was submitted to Loughborough's Institutional Repository (https://dspace.lboro.ac.uk/) by the author and is made available under the following Creative Commons Licence conditions.

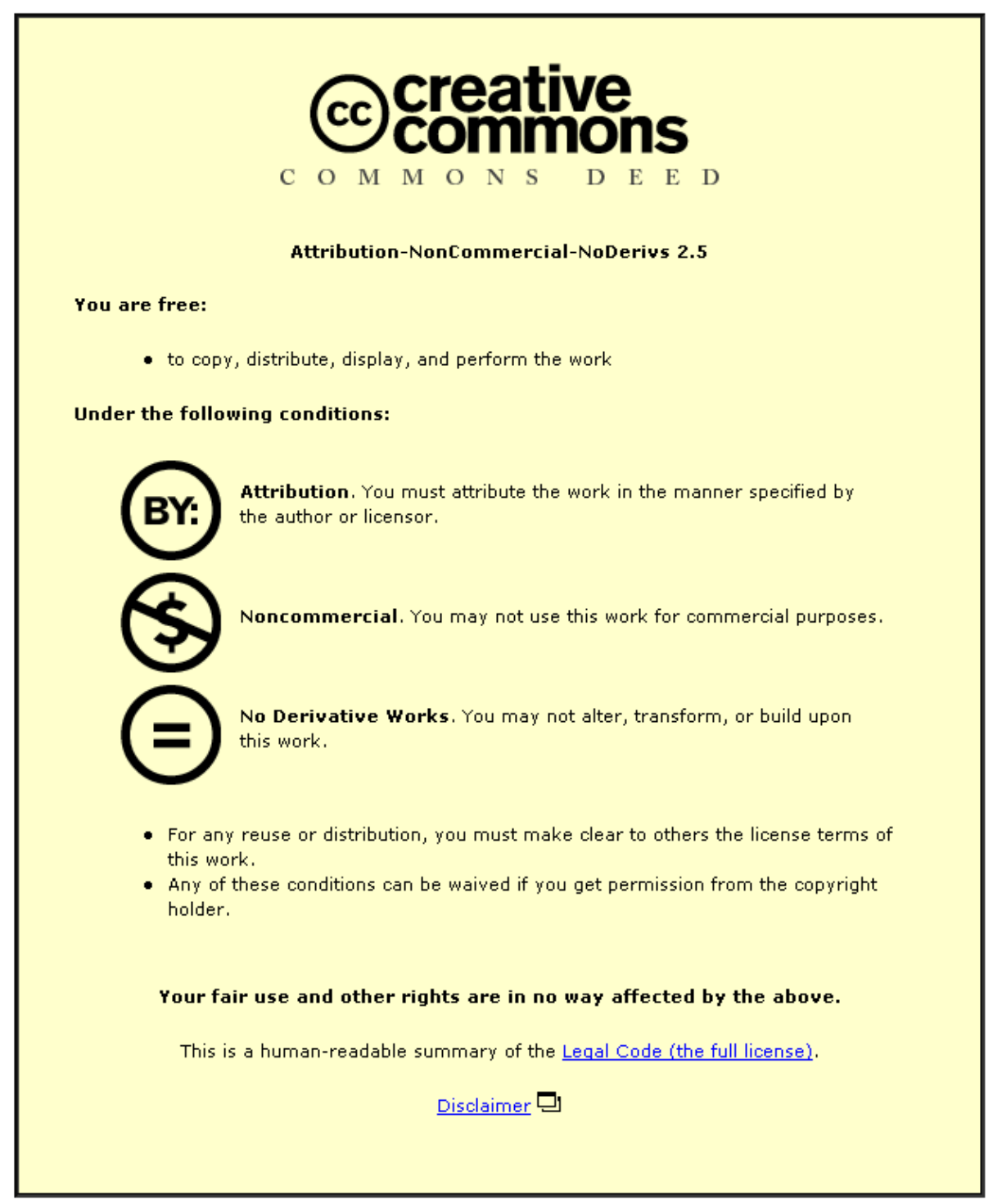

For the full text of this licence, please go to: http://creativecommons.org/licenses/by-nc-nd/2.5/ 


\title{
Partial cancellation of shaking force harmonics by cam modification
}

\author{
S-T Chiou ${ }^{1}$ and T H Davies $^{2}$ \\ ${ }^{1}$ Mechanical Engineering Department, National Cheng-Kung University, Tainan, Taiwan \\ ${ }^{2}$ Department of Mechanical Engineering, Loughborough University, Loughborough, Leicestershire
}

\begin{abstract}
An optimization procedure for minimizing the shaking force harmonics of machinery is described. Because of constraints imposed by the specification, optimization is unlikely to make any significant reduction in the fundamental, machine frequency, term of the shaking force. That term, normally the largest, must be substantially reduced by other means for the procedure described here to be cost effective. Earlier work describing methods of reducing the fundamental term are cited. An extrusion press machine incorporating two planar mechanisms, one crank driven and one cam driven, provides an example. Two designs of the cam-driven mechanism, referred to here as cams 1 and 2, are in use in installed extrusion press machines. Harmonic analysis of the shaking force as a function of time shows that cam 2 produces smaller amplitudes of shaking force harmonics. The ten variables used in the optimization process are the amplitudes and angles of the first five terms of a trigonometric series defining the acceleration of a reciprocating mass driven by a hypothetical third cam, cam 3 , which is similar to cam 2 but stripped of all harmonics of order higher than the fifth. The objective function is the sum of the amplitudes of the horizontal components of the second to fifth shaking force harmonics on the machine as a consequence of accelerations of the moving parts of both mechanisms. Several equality and inequality constraints must be met. For the extrusion press machine the result is a reduction in the amplitudes that would be created if cam 3 were to be used by approximately 10, 75, 65 and 100 per cent respectively. The improvement over cam 1 is greater. The procedure is particularly suited as a retrofit measure where the number of installed machines is large in relation to the future rate of production of new machines: a cam that has been removed from one machine can be re-cut for use on another machine.
\end{abstract}

Keywords: balancing, cam, harmonic, mechanism, optimization

\section{NOTATION}

$a_{0} \quad$ constant term used in formulae for the displacement of bearing I provided by cams 3,4 and 5

$a_{k} \quad$ half the coefficents of a finite $(1 \leqslant k \leqslant 5)$ trigonometric series, defining the acceleration of bearing I provided by cams 3,4 and 5; also the magnitudes of each of the equal contra-rotating acceleration vectors, the resultant of which represents those coefficients

$F_{k} \quad$ half the amplitude of the $k$ th-order frequency term of the horizontal component of the shaking force for the whole machine; also the magnitude of each of the equal

The MS was received on 10 August 1995 and was accepted for publication on 30 December 1996. contra-rotating force vectors, the resultant of which represents that term
$h_{k}, v_{k}$
$I_{j}$
$m_{j}$
$t$
$u$
$X_{\mathrm{I}}, V_{\mathrm{I}}, A_{\mathrm{I}}$
$2 a_{k} \cos \theta_{k}$ and $2 a_{k} \sin \theta_{k}$; variables used in the optimization of cam 4
centroidal mass moment of inertia of member numbered $j$
mass of member numbered $j$
time
normalized variable used in formulae for $X_{\mathrm{I}}, V_{\mathrm{I}}$ and $A_{\mathrm{I}}$, the motion equations of bearing I, provided by cam 1

$y$ horizontal displacement of bearing labelled I and its first and second time derivatives, namely velocity and acceleration function of $\theta$ used in the motion equations of bearing I provided by cam 2

$\theta, \omega$ driveshaft angular displacement from the start of the cycle and its first time derivative, namely angular velocity 
$\theta_{k} \quad$ angular displacements from the horizontal, at the start of the cycle, of two contra-rotating vectors of equal magnitude representing the horizontal component of the $k$ th-order frequency term of acceleration of shaking force

\section{INTRODUCTION}

The paper is concerned with the balancing of planar machinery incorporating two or more mechanisms driven synchronously, at least one of which is cam operated. The synchronization can be mechanical whereby cranks and cams are all driven from a single driveshaft either directly or through gearing, or driven by independent electronically synchronized drives. A further restriction is that all mechanisms share a central plane of symmetry so that the resultant unbalanced forces lie in that plane and there are no unbalanced moments with components parallel to that plane. Should this not be so then any such moment components are ignored.

As bibliographies $(\mathbf{1}, \mathbf{2})$ on the subject of balancing testify, most attention has been paid to achieving partial balance by changing the shape of moving parts, hitherto determined from considerations of strength and stiffness alone, in order to relocate their centres of mass without affecting their kinematic function. This inevitably results in moving parts having a greater mass than they would otherwise have. Consequently, there is a trade-off between reduced shaking force, on the one hand, and increased amplitudes of driving torque harmonics and bearing loads, on the other. Furthermore, if, as is usual, the dimensions and angles determining kinematic performance are chosen first, the design process becomes an iterative one, often with disappointing results. For example, because the balanced set of moving parts imposes greater bearing loads than they would in an unbalanced design, those bearings and their housings need to be larger, and so more massive, than they would otherwise be, and this in turn requires members of greater size and mass to support them.

To avoid increased bearing loads and driveshaft torque harmonics, balance weights can be restricted to shafts that rotate at constant speed. Chiou and Davies (3) represent the shaking force and moment as a series of frequency terms. They show that a frequency term of order $k$ can be represented by a pair of contra-rotating, generally unequal, force vectors rotating at angular velocity $k \omega$, where $\omega$ is the driveshaft angular velocity. Furthermore, the shaking moment about the origin is also represented correctly if these force vectors both rotate about a fixed centre $C_{k}$, a location that is an invariant of the machine. To balance such forces it is more convenient to use balance masses integral with two shafts rotating about different centres: a generalized Lanchester balancer for both forces and moments of one frequency. In reference (3) it is shown that either of these centres can be chosen arbitrarily and methods are provided for finding the location of the other centre once the arbitrary choice of location for one has been made. The magnitude of the first-order frequency term will almost always be the largest. There is usually space on the driveshaft itself to carry a balance mass that can completely or partially balance the shaking force vector that rotates in the same sense as the driveshaft. If the unbalanced shaking force vector that rotates in the same sense as the driveshaft can be substantially balanced in this way only one additional shaft is required - one that carries a balance mass to cancel the unbalanced shaking force vector that rotates in the sense contrary to that of the driveshaft. Using the information provided in reference (3) Davies and Niu (4) explain several ways of balancing the shaking force and moment of cycle frequency as a retrofit measure for installed machinery.

Added shafts carrying rotating masses to balance shaking force frequency terms that are harmonics of cycle frequency are less likely to be cost effective. Firstly, this is because the amplitudes of those rotating forces are smaller than those of the fundamental yet the costs of the retrofit are comparable. Secondly, unless provision has been made at the design stage, there are unlikely to be existing shafts rotating at exact multiples of driveshaft cycle frequency so that two additional shafts are needed as a retrofit measure. However, despite the amplitudes of shaking force harmonics being smaller than those of the fundamental, their importance becomes of greater significance if the shaking force of cycle frequency is balanced in the manner described in references (3) and (4). This is because the benefit of balancing only the first frequency term is gained from the higher production rate that can be achieved by using a higher operating speed than is possible without such a balancing measure. This higher production could be called the speed dividend. The new higher operating speed might be limited by heat generation, bearing life or internal vibration. Another possibility is that the new operating speed will be limited by the increased amplitudes of shaking force and moment of frequency terms of higher order than the fundamental cycle frequency, the harmonics of that frequency. These amplitudes will have increased because they are proportional to the square of the operating speed. It is this possibility that is the concern of this paper.

\section{NUMERICAL OPTIMIZATION}

It is usual for the kinematic specification of the mechanism to prescribe the strokes of output members and the maximum angular or translational displacements of those members. It is also usual for the first-order frequency term of the displacement and its time derivatives to predominate. If the masses of these output members 
are also large then it is unrealistic to expect much, if any, reduction in the fundamental frequency term of the shaking force by using that term in the objective function to be minimized, either alone or in combination with high-order terms.

Furthermore, the experience of the authors is that there is also little scope for optimization to reduce shaking force harmonics attributable to crank-driven linkage mechanisms. They believe that this is because, for a linkage mechanism, the number of design variables such as distances between bearings that affect kinematic performance is finite, indeed small. The kinematic aspects of the specification impose equality and inequality constraints that, in effect, reduce this number further. Also, for linkage mechanisms, the hyper-space occupied by these design variables does not correspond closely with the hyper-space of the variables from which the objective function to be minimized is formulated. Thus a set of dimensional changes, constrained to satisfy the equality and inequality constraints of the specification, does not necessarily make a significant change in the amplitudes of the frequency terms of the shaking force. Put another way, the variables may be ill-conditioned for the optimization of the objective function.

In two respects important to this paper some camdriven mechanisms differ from linkage mechanisms. If the displacement of the part driven by the cam can be expressed throughout the cam cycle by a continuous trigonometric series then the acceleration of that part can also be expressed by a trigonometric series. These acceleration coefficients are exactly proportional to the frequency terms of the shaking force that the part creates by its motion. They are perfectly conditioned, therefore, for the purpose of minimizing shaking force harmonics. A cam mechanism driving a part having a motion expressed by a trigonometric series also differs from a crank-driven mechanism in that there are an infinite number of variables, the coefficients of the series, that could be used as design variables in an optimization procedure. In practice, of course, numerical optimization can only accommodate a finite number of such variables and the optimization proceeds most efficiently when this number is small. The first few terms of the series are suitable as generally they are of largest amplitude but there is no upper limit to the number that could be used except for computational costs. If the output member is driven indirectly by the cam through one or more intermediate links then the contribution those intermediate members make to the shaking force harmonics is clearly related to the trigonometric series expressing the acceleration of the output member they drive. Thus while the acceleration coefficients and angles defining the series are not perfectly conditioned for altering the shaking force harmonics attributable to those intermediate parts, they are nonetheless well-conditioned.

However, the objective is not to minimize the contrarotating force vectors representing the shaking force harmonics attributable to the cam mechanism. Instead, it is to so modify those vectors that they become, as near as possible, equal and opposite to those attributable to the crank-driven mechanism, thereby causing partial cancellation.

Ideally the appropriate time for such considerations is at the design stage, but there are also attractions in uprating (i.e. increasing the speed of) installed machinery at low cost. Numerical optimization has been used to try to reduce the shaking force harmonics of installed extrusion press machines that are now described.

\section{THE EXTRUSION PRESS MACHINE}

This machine has been described elsewhere $(\mathbf{5}, \mathbf{6})$ in greater detail than is required here. For ease of reference Fig. 1 shows, schematically, the components of the crank-driven and cam-driven mechanisms, together with relevant dimensions, and the masses and moments of inertia of moving parts. The crank-driven mechanism drives a reciprocating ram, 5 , that causes products to be redrawn and then extruded. The cam-driven mechanism also drives a reciprocating part, 8, that holds down the product during redraw. The only contribution to unbalance not considered is the mass eccentricity of the cam itself. The cam makes a small contribution to the primary shaking force that can be eliminated by the use of a primary Lanchester balancer; it has no effect on shaking force harmonics, the subject of this paper, and is ignored.

As explained in Section 1, by using FFT (fast Fourier transform) analysis, the variable shaking force vector can be represented throughout the cycle by frequency terms. The frequency term of order $k$ can be represented in turn by two force vectors, each of constant magnitude, contra-rotating at a constant speed that is a multiple $k$ of the driveshaft angular velocity $\omega$. The information required to define these vectors comprises two force magnitudes and two angles. These angles are those subtended by the force vectors to the horizontal at the start of the cycle. Simplification results from confining attention to horizontal components of unbalance because the horizontal components of each frequency term can be expressed by two equal contra-rotating force vectors having mirror symmetry about the horizontal. This reduces the information content to one amplitude and one angle.

There is justification for confining attention to the horizontal components of the shaking force for the extrusion press machine. The output members 5 and 8 reciprocate horizontally, the centres of mass of members 3, 4, 6 and 7 have only a small displacement vertically and although the centres of mass of members 1 and 2 have a large vertical displacement this is predominantly, and for member 1 entirely, of first-order frequency. As 


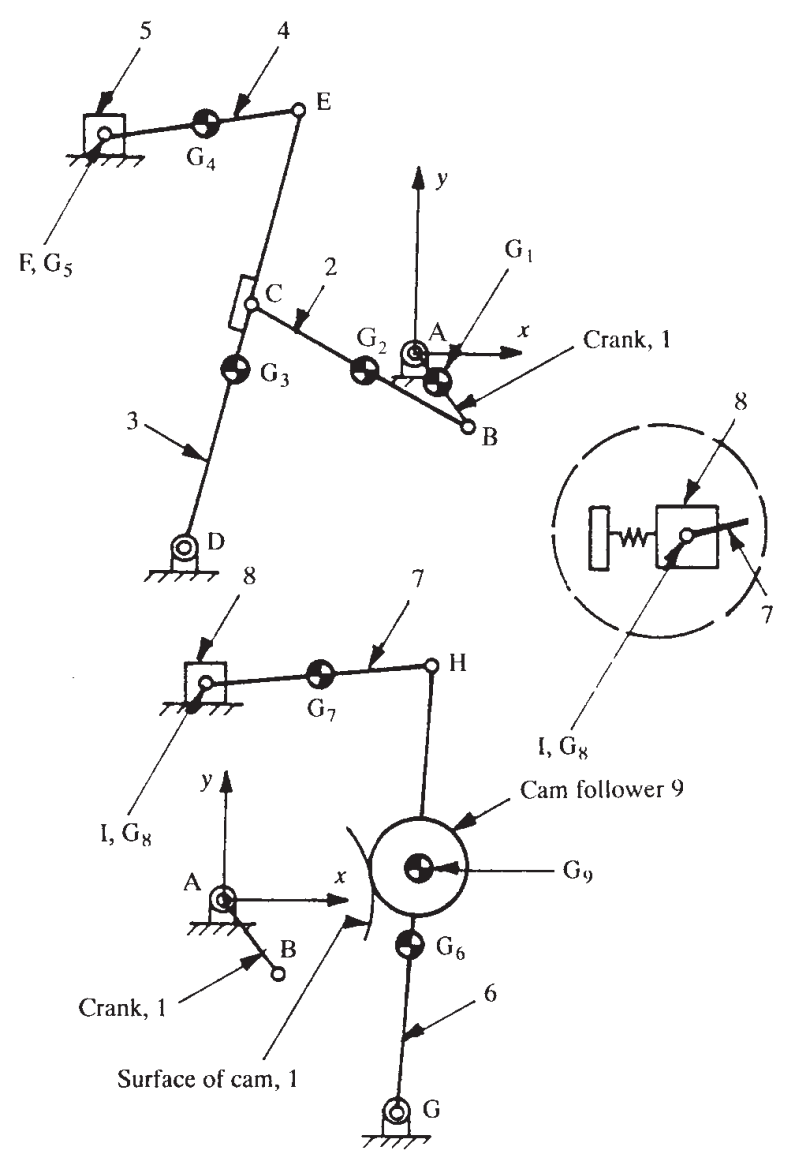

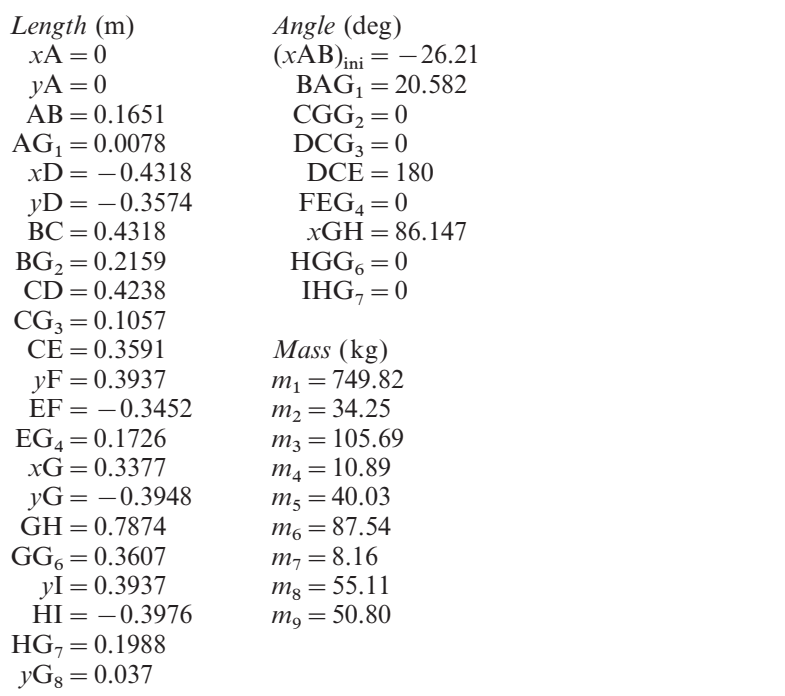

Fig. 1 The crank- and cam-driven mechanisms of an extrusion press machine, relevant dimensions and the masses of moving parts. Note that the angle $(\mathrm{XAB})_{\mathrm{ini}}$ is correct only for cam 1 ; for cam 2 it is $-53^{\circ}$ explained earlier, first-order unbalance must be balanced by other means.

To avoid unnecesary computational effort it is desirable to identify at the outset the contribution to the horizontal components of the shaking force made by parts driven by the crank, because this remains unchanged throughout all subsequent optimizations. This contribution is found by setting the masses and moments of inertia of the parts driven by the cam to zero. The frequency spectrum for the crank-driven mechanism (Fig. 2) remains unchanged whatever cam is used. It is typical of a linkage mechanism that high-order terms are absent; only the first five order terms are significant.

Attention is now focused on the cams and the parts they drive. There are two cams in use in existing installed machinery; they are referred to here as cam 1 and cam 2 . The motion they create is described next.

\subsection{Cam 1: modified trapezoidal}

The formulae that determine the horizontal motion of bearing I, a fixed point on the output member 8 of the cam-driven mechanism, are provided in the Appendix. The displacement, velocity and acceleration of bearing I for machines in which cam 1 is used are plotted in Fig. 3.

The contribution attributable to the cam-driven mechanism is found by setting the mass and moment of inertia of members 1 to 5 to zero during FFT analysis. The shaking force hodographs for the two mechanisms are provided in reference (6); they are not shown here. The hodograph of the cam mechanism is nearly a straight horizontal line, for reasons given above, with many overlaid points during the long dwell periods. The maximum horizontal inertia force due to accelerations of members

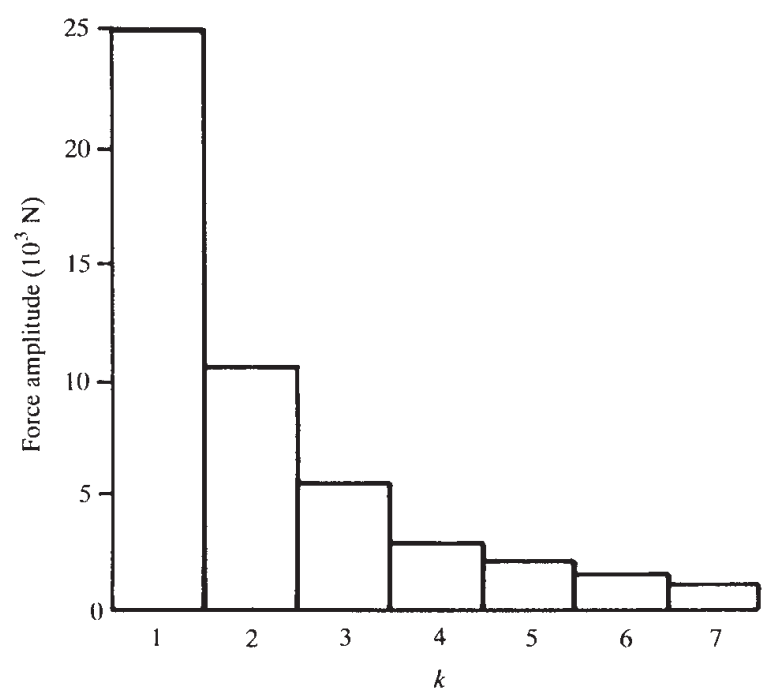

Fig. 2 The frequency spectrum of the horizontal component of the shaking force for the crank-driven mechanism only 

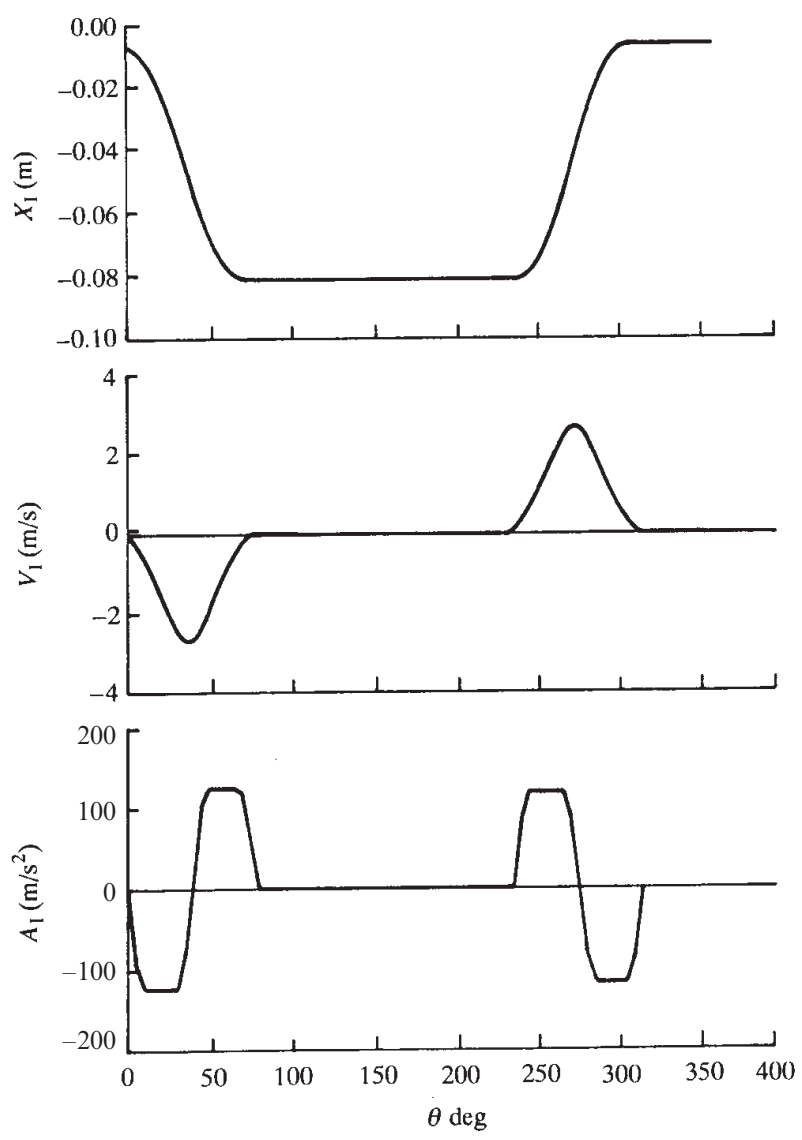

Fig. 3 The motion of bearing I on sliding member 8 using cam 1

6, 7 and 8 is about $16300 \mathrm{~N}$. Because existing parts are designed for this force it is important that any new cam should not be subjected to higher loads.

In contrast to the crank-driven mechanism, the frequency spectrum for the mechanism driven by cam 1 is rich in high-frequency terms, as Fig. 4 shows. It is desirable to reduce these by using a cam providing continuous motion without dwells. Another cam, referred to here as cam 2, also in use on some installed machines, does this by employing lost motion and an air-operated spring. Cam 2 is described next.

\subsection{Cam 2: a continuous motion cam}

Figure 1 and the accompanying data remain relevant for cam 2 with the exception that angle $(\mathrm{XAB})_{\text {ini }}$ is $-53^{\circ}$ instead of $-26.21^{\circ}$. The displacement of bearing I and its time derivatives are plotted in Fig. 5. The maximum shaking force is reduced to $8000 \mathrm{~N}$ and Fig. 4 shows that the frequency spectrum is a substantial improvement on that of cam 1. The motion of member 8 provided by cam 2 is expressed by two functions and there is a discontinuity in the slope of the acceleration curve at the

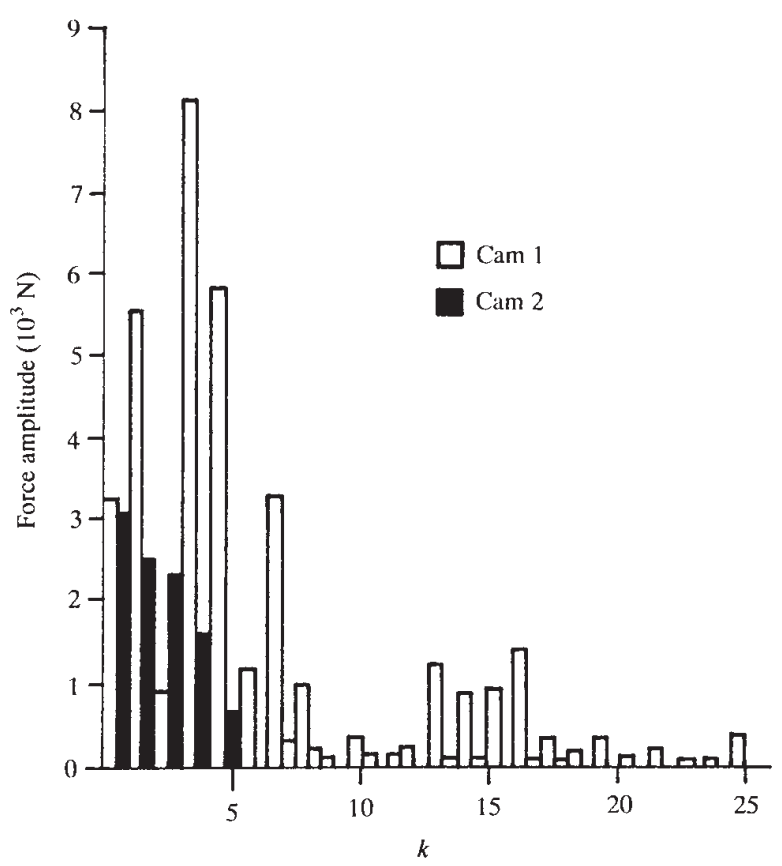

Fig. 4 Frequency spectra of the horizontal components of the shaking force for the mechanisms driven by cams 1 and 2
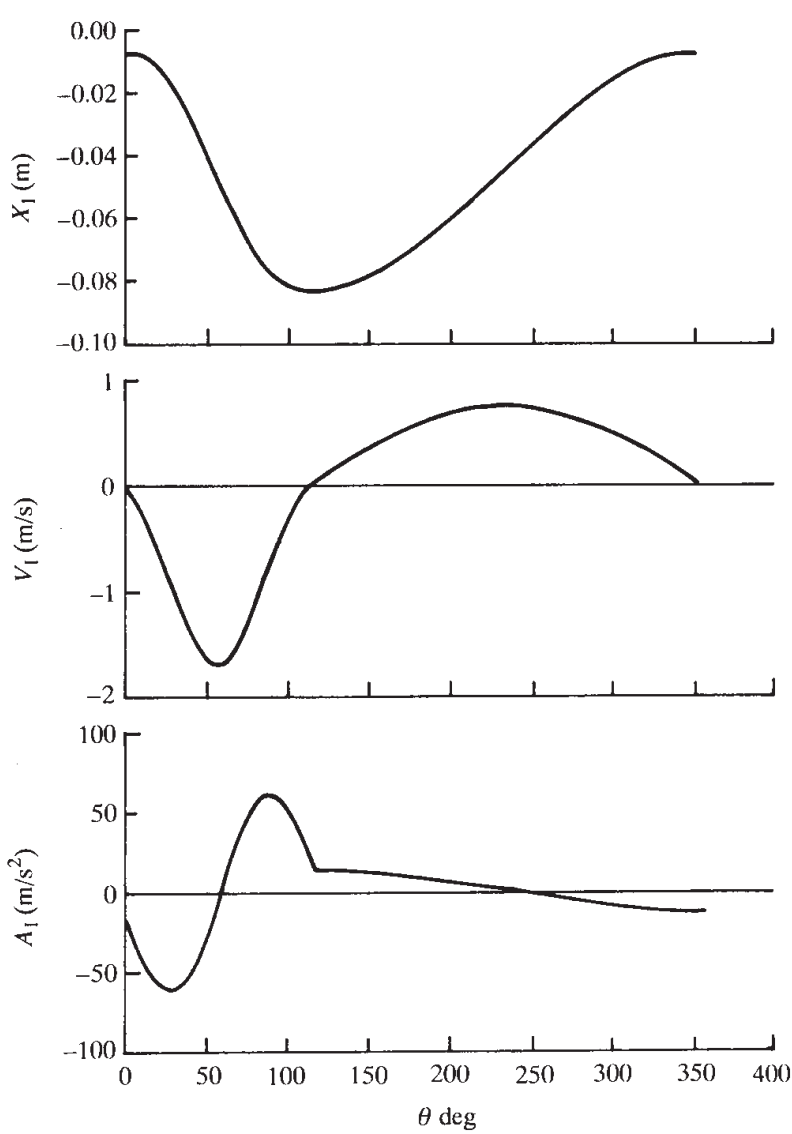

Fig. 5 The motion of bearing I using cam 2

Proc Instn Mech Engrs Vol 211 Part C 
instant when one function is replaced by the other. Instead, a continuous trigonometric function is required, and this is provided by the Fourier series for the motion of bearing I found from FFT analysis. As Fig. 4 shows, like the crank-driven mechanism, only the first five terms are significant.

It was judged that a suitable objective function for optimization would be to minimize the second- to fifthorder terms, usually referred to as the first to fourth harmonics. It should be remembered that the intention was to drastically reduce or eliminate the first-order (fundamental) term by use of a generalized Lanchester balancer.

Initial values for such an optimization are needed. Instead of using cam 2 for this purpose a very similar cam, cam 3, was conceived, which provides bearing I with a motion expressed by frequency terms of order 1 to 5 only.

\subsection{Cam 3: a truncated trigonometric series}

The first step was to truncate the series defining the acceleration of member 8 provided by cam 2 to leave the first five terms. Because higher order terms are discarded the displacement of I is not exactly that provided by cam 2 . In particular, cam 2 meets the requirement that

$$
X_{\mathrm{I}}=-0.0069 \mathrm{~m} \quad \text { and } \quad V_{\mathrm{I}}=0
$$

when $\theta=0^{\circ}$ (bottom dead centre)

and

$$
\begin{aligned}
& X_{\mathrm{I}}=-0.00831 \mathrm{~m} \text { and } V_{\mathrm{I}}=0 \\
& \text { when } \theta=120^{\circ} \text { (top dead centre) }
\end{aligned}
$$

where $X_{\mathrm{I}}, V_{\mathrm{I}}$ are the displacement and velocity respectively of bearing $\mathrm{I}$ and $\theta$ is the crank angular displacement from the start of the cycle.

By leaving the truncated series for cam 2 unadjusted the error is insignificant, but it was deemed to be essential that equality constraints should be met exactly during the optimization procedures that follow. To meet these equality constraints the constant term and the amplitudes of the first two frequency terms of the series were altered very slightly to meet these requirements. The outcome is cam 3, with bearing I motion plotted in Fig. 6. By comparing Figs 5 and 6 it is seen that the main effect of truncation is to remove the abrupt change in the slope of the acceleration curve for cam 2, which is evident in Fig. 5. The maximum difference in the displacement curves is $0.356 \mathrm{~mm}$ and the corresponding difference in cam radius is $0.195 \mathrm{~mm}$.

Numerical optimization was then used to reduce the second- to fifth-order terms of the horizontal shaking force component for the whole machine. To illustrate the strategy graphically, Fig. 7 shows vector triangles for
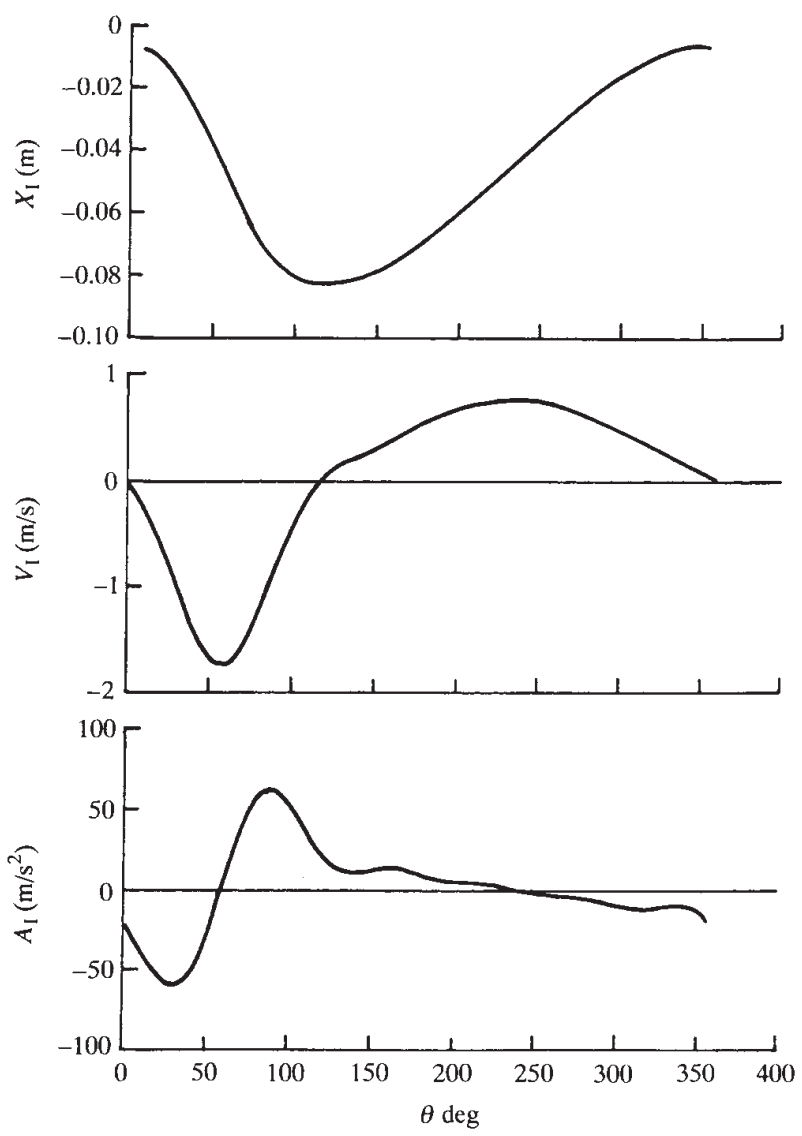

Fig. 6 The motion of bearing I using cam 3

the second to fifth frequency terms. Each vector of the triangle is one of two vectors of equal magnitude that have mirror symmetry about the horizontal and contrarotate at angular velocity $k \omega$. The vector sum of each of these pairs is a horizontal vector representing the horizontal component of the shaking force at the start of the cycle. The sides of each triangle represent the contributions made by the crank-driven mechanism, the cam-driven mechanism using cam 3 and both mechanisms.

The objective of optimization is to alter the vectors representing the contribution made by the cam-driven mechanism so that, for $k=2, \ldots, 5$, those vectors (fine full lines in Fig. 8) are, as near as possible, equal and opposite to the vectors (dashed lines) that are attributable to the crank-driven mechanism. The consequence is that the magnitudes of their resultants (thick full lines) are minimized. These resultants, of magnitude $F_{k}$, when added to their equal partners having mirror symmetry about the horizontal, are the horizontal components of shaking force of order $k=2, \ldots, 5$. At the start of the cycle the magnitude of the horizontal component of order $k$ is $2 F_{k} \cos \theta_{k}$.

Ideally, the authors would have wished to reduce $F_{k}$ to zero for each order $k=2,3,4,5$, but numerical 

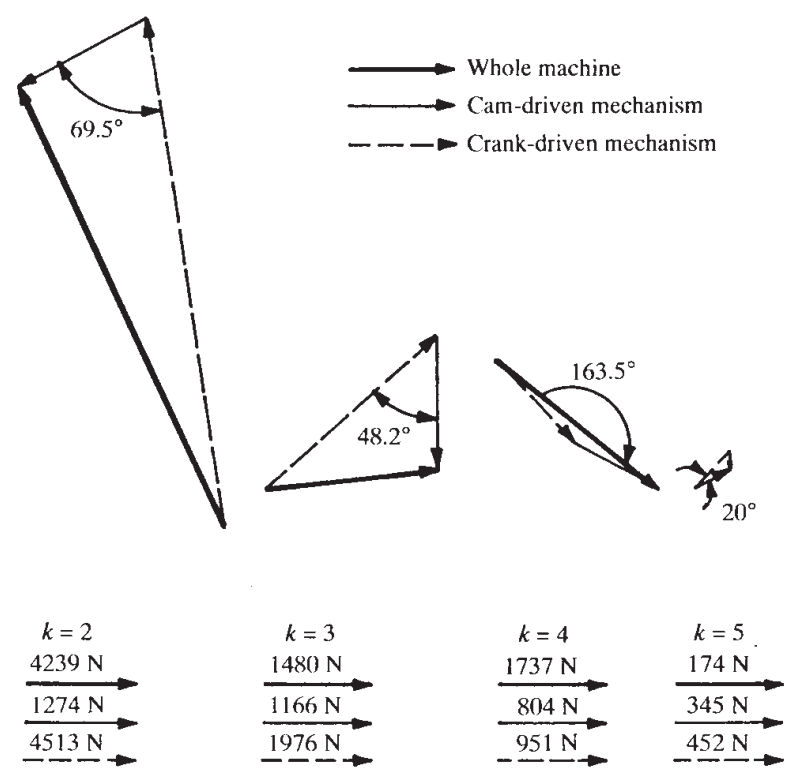

Fig. 7 Vector triangles for the second to fifth frequency terms of the horizontal component of the shaking force using cam 3. Each term is represented, at the start of the cycle, by two equal contra-rotating force vectors symmetrically disposed about the horizontal; only one of each pair is shown

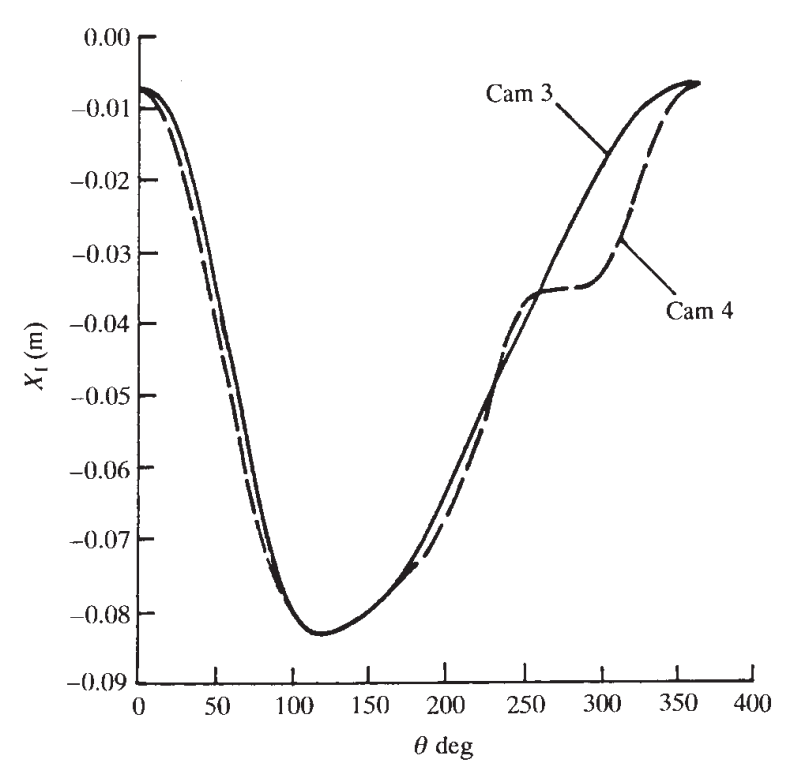

Fig. 8 Displacement curves of bearing I using cams 3 and 4

optimization can only minimize one function and these are four independent functions. We therefore chose to minimize $\Sigma F_{k}, k=2,3,4,5$, thereby implicitly giving each of the four terms an equal weighting. Other weightings were subsequently tried but the results were judged to be inferior.

\subsection{Cam 4: the result of the first optimization}

The following is a summary of the objective function, the variables, their initial values and the constraints placed on them.

Objective function:

$$
\text { Minimize } \sum_{k=2}^{5} F_{k}
$$

where $F_{k}$ is half of the amplitude of the $k$ th-order term of the horizontal component of the shaking force for the whole machine.

Variables:

$$
\begin{gathered}
v_{k}=2 a_{k} \sin \theta_{k}(k=2,3,4,5) \\
h_{k}=2 a_{k} \cos \theta_{k}(k=3,4,5)
\end{gathered}
$$

where $2 a_{k}$ and $\theta_{k}$ are the terms of the trigonometric series representing $A_{\mathrm{I}}$. The remaining four variables, $h_{1}, h_{2}, v_{1}$ and $a_{0}$ (the constant), are used to satisfy the four equality constraints stated in Section 3.3 when $\theta=0^{\circ}$ and $120^{\circ}$.

\section{Initial values:}

The values of $2 a_{k}$ and $\theta_{k}$ for cam 3 are given in the Appendix.

$$
\begin{array}{ll}
\text { Inequality constraints: } & \\
V_{\mathrm{I}} \leqslant 0 & \text { while } 0^{\circ} \leqslant \theta \leqslant 120^{\circ} \\
V_{\mathrm{I}} \geqslant 0 & \text { while } 120^{\circ} \leqslant \theta \leqslant 360^{\circ}
\end{array}
$$

where $\theta$ is the angular displacement of the cam from the start of the cycle.

The outcome of optimization is cam 4, with a new truncated series for the displacement of bearing I and the time derivatives presented in the Appendix. The displacement of bearing I using cams 3 and 4 are compared in Fig. 8. Further details of cam 4 are provided in reference (6) but not here because cam 4 proved to be unacceptable. The fault was drawn to the attention of the authors by industrial collaborators. It was that the displacement of bearing I should not be less than $-0.01852 \mathrm{~m}$ when the cam displacement angle is $35^{\circ}$. As Fig. 8 shows, during the interval $0<\theta<120^{\circ}$, while the cam follower is falling, the displacement of bearing I is smaller using cam 4 than it is using cam 3 and the requirement is not met.

This then was an omission from the specification that had originally been assumed. It was necessary therefore to include a new inequality constraint. The opportunity was taken to review also the role of the four equality and two inequality constraints used in the first optimization. It was concluded that the four equality constraints are unnecessarily restrictive and that the specification can be met without them, thereby enabling $v_{1}, h_{1}$ and $h_{2}$ to be available as variables.

Another consequence of the review was that the two inequality constraints are amended and three further 
ones are added that guarantee satisfactory performance. The second inequality constraint listed below corrects the faults mentioned above.

\subsection{Cam 5: the result of the second optimization}

The following is a summary of the objective function, the variables, their initial values and the constraints placed on them.

Objective function:

$$
\text { Minimize } \sum_{k=2}^{5} F_{k}
$$

where $F_{k}$ is half of the amplitude of the $k$ th-order term of the horizontal component of the shaking force for the whole machine.

Variables:

$$
\begin{aligned}
& v_{k}=2 a_{k} \sin \theta_{k}(k=1,2,3,4,5) \\
& h_{k}=2 a_{k} \cos \theta_{k}(k=1,2,3,4,5)
\end{aligned}
$$

and $a_{0}$, the constant, is used to satisfy the equality constraint

$$
\left(X_{\mathrm{I}}\right)_{\min }=-0.083109 \mathrm{~m}
$$

Initial values:

The values of $2 a_{k}$ and $\theta_{k}$ for cam 4 are given in the Appendix.

The single equality constraint is satisfied by finding the lowest three values of $X_{\mathrm{I}}$ generated, using a quadratic polynomial approximation to calculate $\left(X_{\mathrm{I}}\right)_{\min }$, and then by altering the value of $a_{0}$ to satisfy the equality constraint.

\section{Inequality constraints:}

$$
\begin{array}{ll}
X_{\mathrm{I}}+0.006 \leqslant 0 & \text { while }-30^{\circ} \leqslant \theta \leqslant 30^{\circ} \\
X_{\mathrm{I}}+0.01852 \leqslant 0 & \text { while } \theta=35^{\circ} \\
X_{\mathrm{I}}+0.08167 \leqslant 0 & \text { while } 105^{\circ} \leqslant \theta \leqslant 135^{\circ} \\
V_{\mathrm{I}} \leqslant 0 & \text { while } 35^{\circ} \leqslant \theta \leqslant 105^{\circ} \\
V_{\mathrm{I}} \leqslant 0 & \text { while } 145^{\circ} \leqslant \theta \leqslant 325^{\circ}
\end{array}
$$

The outcome is cam 5, providing bearing I with the motion given in the Appendix and plotted in Fig. 9.

\section{DISCUSSION}

The benefits of optimization can be judged by comparing the second to fifth terms of the frequency spectrum for cams 3 and 5, shown in Fig. 10. While substantial reductions are made in the amplitudes of the third, fourth and fifth terms the reduction in the second term is limited to 10 per cent and, as anticipated, there is no change in the fundamental term. The triangle of force
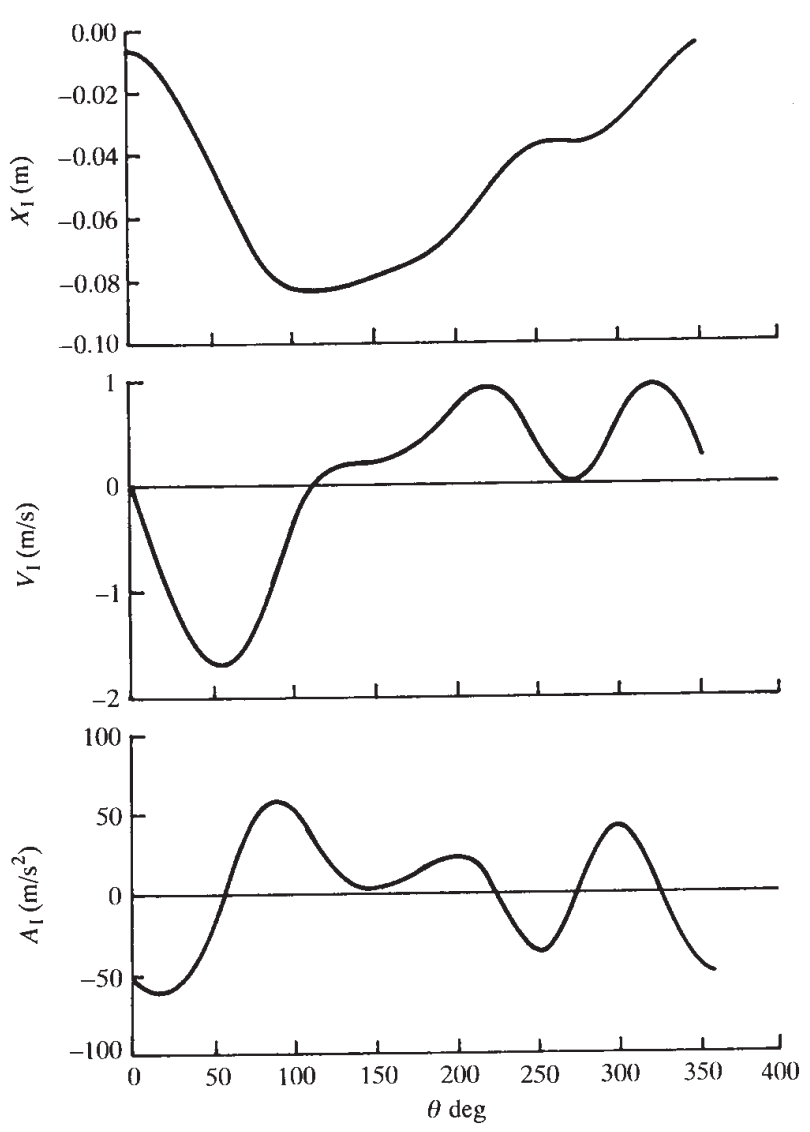

Fig. 9 The motion of bearing I using cam 5

vectors for cam 5 is shown in Fig. 11, which should be compared with Fig. 7.

Some alternative objective functions were tried but the results were always worse. For example, the objective function

$$
\sum_{k=2}^{5}\left(k^{2}\right) F_{k}
$$

gave results that were inferior to those obtained using equal weighting to each frequency term.

The fourth and fifth inequality constraints compelling the displacement of member 8 to be monotonic during forward and backward motion is one that is open to question. It is evident from Figs 9 and 12 that there is a hesitation, but because of those constraints no reversal, at about $\theta=280^{\circ}$. This suggests that, without those inequality constraints, optimization would find a smaller value for the objective function if a reversal in the displacement had taken place. The authors judged that it would be prudent not to allow this.

If the modifications result in loadings on parts that are lower than those for which they are designed, as they are in the example described in this paper, then consideration might be given to allowing the mass of selected parts to be additional variables in the optimization, 


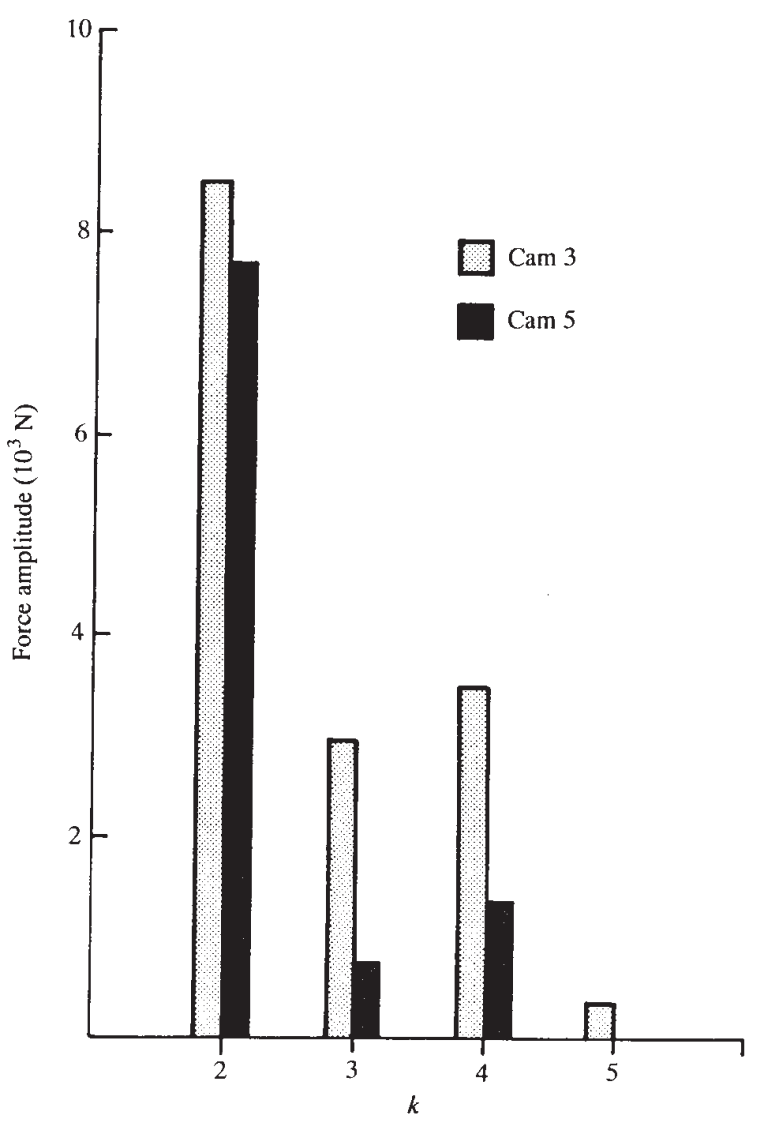

Fig. 10 The frequency spectrum of the horizontal shaking force amplitudes for the whole machine using cams 3 and 5

subject to the inequality constraint that those masses must not be reduced.

\section{CONCLUSIONS}

1. Optimization can only make a substantial improvement if the masses of parts driven by the cam sum to a substantial proportion of the total mass.

2. The kinematic specification, represented by equality and inequality constraints, limits the reduction that can be made to the frequency terms of lower order. In the example shown here this is certainly true of the first-order term and is substantially true of the second-order term.

3. The costs of a retrofit package involves the removal, re-cutting and replacement of a cam. If one new cam is made for the first machine to be modified then the cam that is removed from that machine can be re-cut for the next machine. Thus only one new cam is required to upgrade the performance of a limitless number of installed machines.

4. If cam 1 is to be replaced by cam 5 in an extrusion C08795 ㄷ IMechE 1997

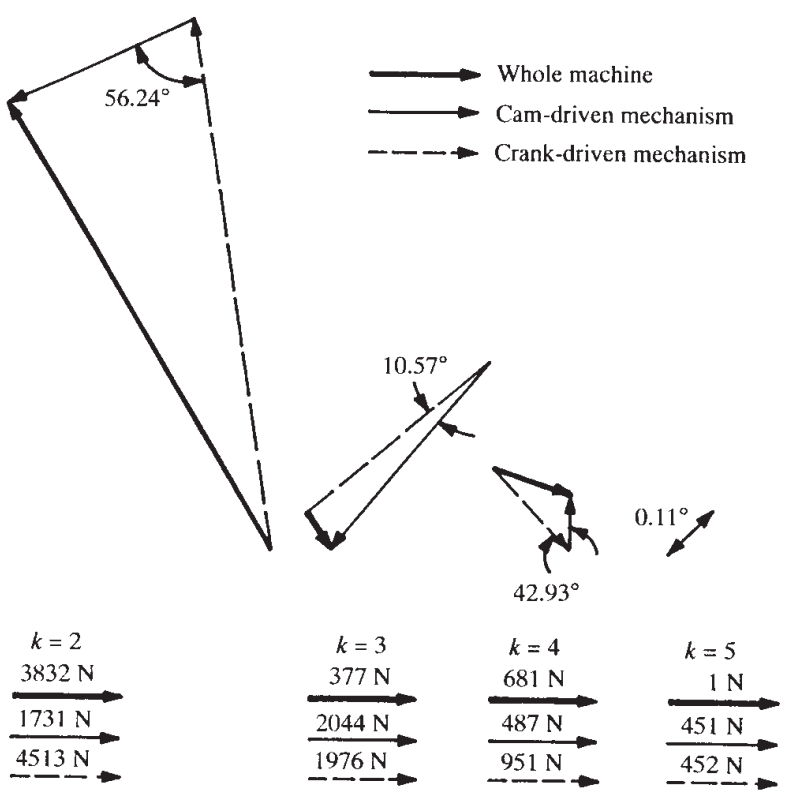

Fig. 11 Vector triangles for the second to fifth frequency terms of the horizontal component of shaking force using cam 5. Each term is represented, at the start of the cycle, by two equal contra-rotating force vectors symmetrically disposed about the horizontal; only one of each pair is shown

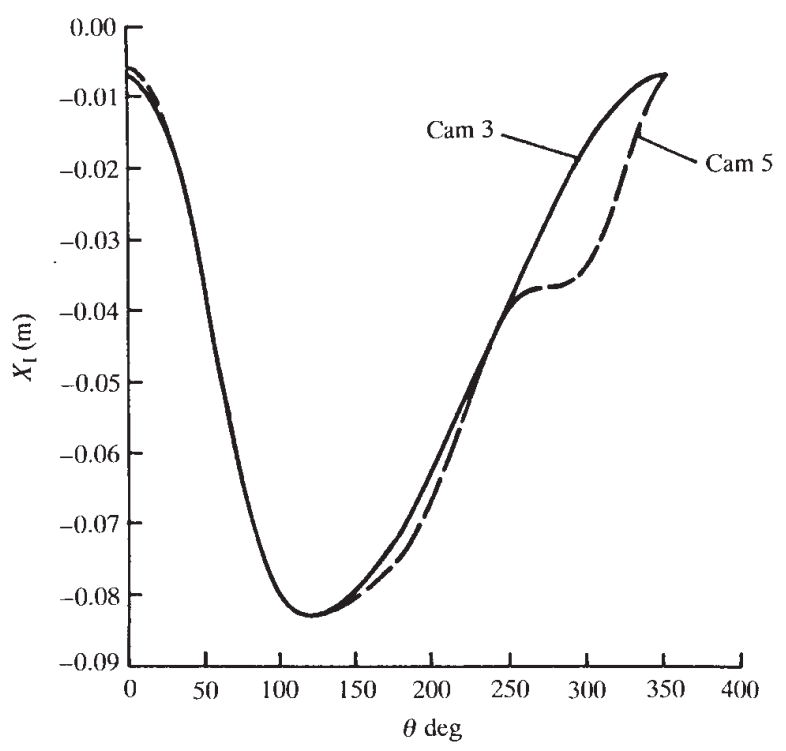

Fig. 12 Displacement curves for bearing I using cams 3 and 5

press machine it is also necessary to install the air spring used in conjunction with cam 2 .

5. The cost effectiveness of a retrofit is difficult to judge without trials because it is not possible to be certain of the greater maximum operating speed that will 
be possible following the improvements that are described in this paper.

\section{REFERENCES}

1 Lowen, G. G. and Berkof, R. S. Survey of investigations into the balancing of linkages. J. Mechanisms, 1968, 3, 221-231.

2 Lowen, G. G., Tepper, F. R. and Berkof, R. S. Balancing of linkages - an update. Mech. Mach. Theory, 1983, 18(3), 213-220.

3 Chiou, S.-T. and Davies, T. H. The ideal locations for the contra-rotating shafts of generalized Lanchester balancers. Proc. Instn Mech. Engrs, Part C, 1994, 208(C1), 29-37.

4 Davies, T. H. and Niu, G. H. On the retrospective balancing of installed planar mechanisms. Proc. Instn Mech. Engrs, Part C, 1994, 208(C1), 39-45.

5 Askari, M. R., Chiou, S.-T. and Davies, T. H. The computer aided design of a retrofit package to reduce the shaking force in a metal can bodymaker machine. In Proceedings of Seminar on High Speed Machinery, 1988, pp. 49-56 (Mechanical Engineering Publications, London).

6 Chiou, S.-T. Studies of retrofit and designs for reducing the shaking force of a can body-making machine. PhD thesis, Loughborough University of Technology, 1988.

\section{APPENDIX}

\section{Motion equations for bearing I on member 8}

There are five cams mentioned in the paper that drive member 8. The motion equations for each are summarized below.

\section{Cam 1}

The following are normalized equations describing the modified trapezoidal law for both the forward and backward motions which are separated by two dwell periods:

Interval

Displacement

Velocity

Acceleration

$\begin{array}{lccc}0 \leqslant u<0.125 & K\left(u-\frac{\sin 4 \pi u}{4 \pi}\right) & K(1-\cos 4 \pi u) & 4 \pi K \sin 4 \pi u \\ 0.125 \leqslant u<0.375 & K\left[2 \pi\left(u-\frac{1}{8}\right)^{2}+u-\frac{1}{4 \pi}\right] & K\left[4 \pi\left(u-\frac{1}{8}\right)+1\right] & 4 \pi K \\ 0.375 \leqslant u<0.625 & K\left[\frac{-\cos 4 \pi\left(u-\frac{3}{8}\right)}{4 \pi}+\pi\left(u-\frac{1}{4}\right)+u\right] & K\left[\sin 4 \pi\left(u-\frac{3}{8}\right)+\pi+1\right] & 4 \pi K \cos 4 \pi\left(u-\frac{3}{8}\right) \\ 0.625 \leqslant u<0.875 & K\left[-2 \pi\left(u-\frac{7}{8}\right)^{2}+u+\frac{1}{4 \pi}+\frac{\pi}{2}\right] & K\left[4 \pi\left(-u+\frac{7}{8}\right)+1\right] & -4 \pi K \\ 0.875 \leqslant u<1.0 & K\left[\frac{\cos 4 \pi\left(u-\frac{7}{8}\right)}{4 \pi}+u-1\right]+1 & -K\left[\sin 4 \pi\left(u-\frac{7}{8}\right)-1\right] & -4 \pi K \cos 4 \pi\left(u-\frac{7}{8}\right)\end{array}$

where $K=2 /(\pi+2)$.

Cam 2

$$
X_{\mathrm{I}}=-0.006909-0.0254 y \mathrm{~m}
$$

where, if $0^{\circ} \leqslant \theta \leqslant 120^{\circ}$,

$$
\begin{aligned}
& y=\frac{3(1-\cos 3 \theta / 2)}{8}+\frac{9}{4}\left(\frac{\theta}{120}-\frac{\sin 3 \theta}{2 \pi}\right) \\
& \frac{\mathrm{d} y}{\mathrm{~d} t}=\left[\frac{9}{16} \sin \frac{3 \theta}{2}+\frac{27}{8 \pi}(1-\cos 3 \theta)\right] \frac{\mathrm{d} \theta}{\mathrm{d} t}
\end{aligned}
$$

$$
\frac{\mathrm{d}^{2} y}{\mathrm{~d} t^{2}}=\left(\frac{27}{32} \cos \frac{3 \theta}{2}+\frac{81}{8 \pi} \sin 3 \theta\right)\left(\frac{\mathrm{d} \theta}{\mathrm{d} t}\right)^{2}
$$

if $120^{\circ} \leqslant \theta \leqslant 360^{\circ}$,

$$
\begin{aligned}
& y=3-\frac{3}{2}\left[1-\cos \frac{3(\theta-120)}{4}\right] \\
& \frac{\mathrm{d} y}{\mathrm{~d} t}=\left[-\frac{9}{8} \sin \frac{3(\theta-120)}{4}\right] \frac{\mathrm{d} \theta}{\mathrm{d} t}
\end{aligned}
$$




$$
\frac{\mathrm{d}^{2} y}{\mathrm{~d} t^{2}}=\left[-\frac{27}{8} \cos \frac{3(\theta-120)}{4}\right]\left(\frac{\mathrm{d} \theta}{\mathrm{d} t}\right)^{2}
$$

with $y$ in inches.

Cam 3

$$
\begin{aligned}
& X_{\mathrm{I}}=-0.045-\sum_{k=1}^{5} \frac{2 a_{k}}{(k \omega)^{2}} \cos \left(k \omega t-\theta_{k}\right) \\
& V_{\mathrm{I}}=\sum_{k=1}^{5} \frac{2 a_{k}}{k \omega} \sin \left(k \omega t-\theta_{k}\right) \\
& A_{\mathrm{I}}=\sum_{k=1}^{5} 2 a_{k} \cos \left(k \omega t-\theta_{k}\right)
\end{aligned}
$$

where

\begin{tabular}{ccc}
\hline$k$ & $2 a_{k}\left(\mathrm{~m} / \mathrm{s}^{2}\right)$ & $\theta_{k}(\mathrm{deg})$ \\
\hline 1 & 23.86 & 150.13 \\
2 & 19.66 & -150.32 \\
3 & 18.00 & -90 \\
4 & 12.41 & -30 \\
5 & 5.33 & 30 \\
\hline
\end{tabular}

\section{Cam 4}

$$
\begin{aligned}
& X_{\mathrm{I}}=-0.048-\sum_{k=1}^{5} \frac{2 a_{k}}{(k \omega)^{2}} \cos \left(k \omega t-\theta_{k}\right) \\
& V_{\mathrm{I}}=\sum_{k=1}^{5} \frac{2 a_{k}}{k \omega} \sin \left(k \omega t-\theta_{k}\right) \\
& A_{\mathrm{I}}=\sum_{k=1}^{5} 2 a_{k} \cos \left(k \omega t-\theta_{k}\right)
\end{aligned}
$$

where

\begin{tabular}{lrr}
\hline$k$ & $2 a_{k}\left(\mathrm{~m} / \mathrm{s}^{2}\right)$ & $\theta_{k}(\mathrm{deg})$ \\
\hline 1 & 21.63 & 150.92 \\
2 & 23.46 & -144.06 \\
3 & 29.62 & -136.36 \\
4 & 10.34 & 25.25 \\
5 & 6.99 & 50.06 \\
\hline
\end{tabular}

\section{Cam 5}

$$
\begin{aligned}
& X_{\mathrm{I}}=-0.048-\sum_{k=1}^{5} \frac{2 a_{k}}{(k \omega)^{2}} \cos \left(k \omega t-\theta_{k}\right) \\
& V_{\mathrm{I}}=\sum_{k=1}^{5} \frac{2 a_{k}}{k \omega} \sin \left(k \omega t-\theta_{k}\right) \\
& A_{\mathrm{I}}=\sum_{k=1}^{5} 2 a_{k} \cos \left(k \omega t-\theta_{k}\right)
\end{aligned}
$$

where

\begin{tabular}{ccr}
\hline$k$ & $2 a_{k}\left(\mathrm{~m} / \mathrm{s}^{2}\right)$ & $\theta_{k}(\mathrm{deg})$ \\
\hline 1 & 21.57 & 155.47 \\
2 & 26.70 & -137.06 \\
3 & 31.55 & -127.55 \\
4 & 7.52 & 90.54 \\
5 & 6.97 & 50.12 \\
\hline
\end{tabular}

